\title{
Genetic Inhibition of sFRP3 Prevents Glial Reactivity in a Mouse Model of Accelerated Aging
}

\author{
Ana Mia Corujo-Ramirez ${ }^{1,2, *}$, Malvika Dua ${ }^{1,3,},{\text { Ki Hyun } \text { Yoo }^{1} \text {, Alfredo Oliveros }}^{1}$, Mi-Hyeon Jang ${ }^{1,4}$ \\ ${ }^{1}$ Department of Neurologic Surgery, Mayo Clinic, Rochester, MN, USA \\ ${ }^{2}$ PREP, Neuroscience Track, Mayo Clinic Graduate School of Biomedical Sciences, Rochester, MN, USA \\ ${ }^{3}$ SURF program, Neuroscience Track, Mayo Clinic Graduate School of Biomedical Sciences, Rochester, MN, USA \\ ${ }^{4}$ Department of Biochemistry and Molecular Biology, Mayo Clinic, Rochester, MN, USA
}

Purpose: Aging is the most significant risk factor for neurodegenerative disorders that are typified by cognitive deficits. Our recent work utilizing BubR1 hypomorphic $\left(B u b R 1^{\mathrm{H} / \mathrm{H}}\right)$ mice, an accelerated aging model, has revealed that genetic inhibition of the endogenous Wnt pathway inhibitor secreted frizzled related protein 3 (sFRP3) plays a neuroprotective role. Neuroinflammation has been suggested as a pathological hallmark of age-related neurodegeneration mediating cognitive impairment. However, whether sFRP3 inhibition has a neuroprotective effect on neuroinflammatory gliosis in $B u b R 1^{\mathrm{H} / \mathrm{H}}$ mice is unknown. Methods: To investigate neuroprotection from aging-related neuroinflammation by sFRP3 in vivo, we generated double Bub $R 1^{\mathrm{H} / \mathrm{H}} ; s f r p 3$ knockout mice and performed immunohistological analysis with cell type-specific markers for astrocytes (glial fibrillary acidic protein), and microglia (ionized calcium-binding adapter molecule 1). Given that the hippocampus is a brain structure critical for learning and memory, and is uniquely affected in aging-related neurodegeneration, we evaluated morphological changes on astrocytes and microglia via confocal imaging.

Results: We demonstrate that $B u b R 1^{\mathrm{H} / \mathrm{H}}$ mice exhibit significantly increased levels of astrogliosis and an increased trend of microglial activation in the hilus and molecular layer of the young adult hippocampus, thus suggesting that BubR1 insufficiency accelerates glial reactivity. Importantly, our results further show that genetic inhibition of sFRP3 significantly recovers the astrogliosis and microglial activation observed in $B u b R 1^{\mathrm{H} / \mathrm{H}}$ mice, suggesting a critical neuroprotective role for $\mathrm{sFRP} 3$ in age-related neuroinflammation.

Conclusions: Our findings suggest that sFRP3 inhibition may represent a novel therapeutic strategy for neurodegeneration.

Keywords: Aging; sFRP3; BubR1 $1^{\mathrm{H} / \mathrm{H}}$ mice; Neuroprotection; Neuroinflammation

- Fund/Grant Support: This work was supported by NIA (R01AG058560, R01CA242158), and Regenerative Medicine Minnesota to M.H.J., NIA (R01AG058560-S1) to A.M.C.R., Mayo Clinic SURF program to M.D., and the Bosarge Family Foundation-Waun Ki Hong Scholar Award for Regenerative Cancer Medicine from the American Association for Cancer Research to A.O.

- Research Ethics: This experimental procedure was approved by the Mayo Clinic Institutional Animal Care and Use Committee (IACUC; \#A1756) in accordance with National Institutes of Health guidelines.

- Conflict of Interest: No potential conflict of interest relevant to this article was reported.

Corresponding author: Mi-Hyeon Jang (D) https://orcid.org/0000-0001-6274-3588 Department of Neurologic Surgery, Mayo Clinic, 200 First Street SW, Rochester, MN 55905, USA

E-mail: jang.mihyeon@mayo.edu

*Ana Mia Corujo-Ramirez and Malvika Dua contributed equally to this study as co-first authors.

Submitted: October 12, 2020 / Accepted after revision: November 5, 2020
This is an Open Access article distributed under the terms of the Creco
commons.org/licenses/by-nc/4.0/) which permits unrestricted non-commercial use, distribution, and reproduction in any medium, provided the original work is properly cited. 


\footnotetext{
- HIGHLIGHTS

- Increased GFAP' astrocyte reactivity is observed in the hippocampus of young BubR $1^{\mathrm{H} / \mathrm{H}}$ mice, suggesting BubR1 insufficiency accelerates astrogliosis.

- BubR $1^{\mathrm{H} / \mathrm{H}}$ mice show an increased trend in $\mathrm{Iba}-1^{+} \mathrm{CD} 68^{+}$activated microglia.

- Genetic inhibition of sFRP3 significantly recovers increased astrogliosis and microglia activation observed in BubR $1^{\mathrm{H} / \mathrm{H}}$ mice.

- sFRP3 inhibition may play a neuroprotective role in age-related neuroinflammation.
}

\section{INTRODUCTION}

Aging is the most significant risk factor for neurodegenerative disorders typified by cognitive deficits, such as Alzheimer disease. Given the persistent rise of the world's elderly population as a result of medical advancements, improving cognitive function is critical to maintaining a healthy and productive elderly population [1]. The mitotic checkpoint kinase BUB-related 1 (BubR1) is encoded by Budding Uninhibited by Benzimidazole $1 \mathrm{~B}$ and is crucial for appropriate chromosome segregation. Mutations in BubR1 can lead to cell aneuploidy, a feature associated with mosaic varied aneuploidy in patients [2,3]. Notably, BubR1 levels progressively decline with natural aging, and mutant BubR1 hypomorphic $\left(B u b R 1^{\mathrm{H} / \mathrm{H}}\right)$ mice that possess reductions in BubR1 levels exhibit accelerated aging phenotypes such as a short lifespan, cataracts, cachectic dwarfism, and sarcopenia $[4,5]$. Recently, our studies have shown that age-related declines in BubR1 impair hippocampal neurogenesis [6], myelination, reduces body and brain size [7], as well as contributes to behavioral and memory dysfunction in mice [8]. These results suggest that reduced levels of BubR1 contribute to age-related neuropathology. Therefore, investigation of molecular targets that can reverse detrimental BubR1-related biological processes is of great interest.

Wnt signaling has emerged as an essential factor in regulating embryonic development, neural stem cell (NSC) maintenance, differentiation, adult neurogenesis, and synaptic plasticity [9]. While Wnt signaling deficits have been shown to lead to impairments in these aforementioned biological processes, dysfunctional Wnt signaling is also associated with aging-related impairments in cognitive function $[10,11]$. We have previously identified the secreted frizzled related protein 3 (sFRP3), a natural antagonist of Wnt signaling, as a critical player that negatively regulates adult hippocampal neurogenesis, the process by which adult-born neurons are generated. Genetic inhibition of sFRP3 stimulates activation of quiescent NSCs, neuronal maturation, neuron production, dendritic growth and dendritic spine formation [12]. These biological processes are associated with promotion of antidepressant action in both mice and humans [13]. However, while enhancing Wnt signaling can ameliorate age-dependent deficits in cellular and memory function [14], whether inhibition of sFRP3 has a neuroprotective role in $\mathrm{Bu}-$ bR1-mediated brain aging remains unknown.

Astrocytes and microglia are glial cells that play essential roles for maintaining healthy brain function [15]. However, in both humans and mice, aging causes abnormal glial function and reactivity, thus impairing their ability to maintain healthy interactions with neighboring brain cells. It is hypothesized that impaired glial function contributes to the inflammatory state observed in aged brains as well as age-related neurodegenerative conditions [16-18]. Although previous research shows that $B u b R 1^{\mathrm{H} / \mathrm{H}}$ mice exhibit accelerated neuroinflammation, including increased astrogliosis and microglia activation $[5,19]$, it remains unknown if Wnt signaling via sFRP3 inhibition, can have a functional impact in age-related neuroinflammation. Therefore, using double $B u b R 1^{\mathrm{H} / \mathrm{H}} ; s f r p 3$ knockout $(\mathrm{KO})$ mice in combination with histological and confocal imaging analysis, we sought to explore whether inhibition of sFRP3 prevents the neuroinflammatory phenotypes observed in $B u b R 1^{\mathrm{H} / \mathrm{H}}$ mice.

\section{MATERIALS AND METHODS}

\section{Mice}

BubR1 hypomorphic $\left(B u b R 1^{\mathrm{H} / \mathrm{H}}\right)$ and sFRP3 KO mice were generated as previously described $[4-6,12] . B u b R 1^{\mathrm{H} / \mathrm{H}}$ mice were backcrossed to the C57BL/6 background for over 10 generations. Adult $B u b R 1$ heterozygous (HET; $B u b R 1^{\mathrm{H} /+}$ ) were crossed to $s f r p$ HET mice to generate BubR1 HET;sfrp3 HET mice. Then, BubR1 HET;sfrp3 HET mice were interbred to BubR1 HET;sfrp3 HET mice to generate BubR1 wild-type (WT);sfrp3 WT mice, $B u b R 1^{\mathrm{H} / \mathrm{H}} ; s f r p 3 \mathrm{WT}$ mice, $B u b R 1^{\mathrm{H} / \mathrm{H}} ; s f r p 3$ HET mice, and $B u b R 1^{\mathrm{H} / \mathrm{H}}: s f r p 3 \mathrm{KO}$ mice [20]. For all experiments, female mice were utilized unless otherwise specified. All groups of mice used were 8-10 weeks of age at the time of initiating experiments and housed in standard cages under a 12-hour light/dark cycle. Standard rodent chow and water were provided ad libitum. Animal care and handling procedures were approved by the Mayo Clinic Institutional Animal Care and Use Committee (IACUC; \#A1756) in accordance with National Institutes of Health guidelines. 


\section{Perfusion Process and Immunostaining}

Mice were perfused at 8-10 weeks of age with $4 \%$ paraformaldehyde (PFA). The brains were kept in 4\% PFA overnight and then switched to $30 \%$ sucrose. Coronal brain sections $(40 \mu \mathrm{m}$ thick) were prepared in serial order across the entire brain using a microtome (Leica SM 2010R; Leica Biosystems Inc., Buffalo Grove, IL, USA) and processed for histological analysis $[6,20]$.

Sets of approximately 4 sections per mouse with the following coordinates (in $\mathrm{mm}$ : posterior $=-2.53$ from Bregma, anterior $=-1.31$ ) from each animal were processed for immunostaining with specific antibodies. To detect astrocytes, we used antimouse glial fibrillary acidic protein (GFAP; 1:500; Cat\# ab53554; Abcam, Cambridge, MA, USA). As a microglial marker we used anti-rabbit ionized calcium-binding adaptor molecule 1 (Iba1; 1:500; Cat\#019-19741; Fujifilm Wako, Tokyo, Japan). To examine microglial activation, we used rat anti-mouse CD68 (1:500; Cat\# MCA1957T; Bio Rad, Hercules CA, USA). For immunostaining with anti-GFAP, an antigen retrieval protocol was performed using a microwave in order to unmask antigens in tissues. Briefly, the citrate buffer (10mM Sodium Citrate, $0.05 \%$ Tween 20, pH 6) was heated for 5 minutes; the sections were placed in the boiling buffer and heated for another 5 minutes at maximum power [6,21]. The buffer was washed off in $1 \times \mathrm{PBS}$ and the sections were incubated overnight with antiGFAP. The sections were incubated with secondary conjugated antibodies Cy3 and Cy5 (1:500; Jackson Immuno Research Laboratories, West Grove, PA, USA). DAPI (Cat\# D1306, Thermo Fisher, Waltham MA, USA) was used for counterstaining.

\section{Confocal Imaging and Analysis}

Z-stack images of the hippocampus were acquired on a Zeiss LSM 780 single-photon confocal system using $n=4$ sections of each genotype. In order to count the number of astrocytes and microglia in the molecular layer and dentate hilus, images were acquired by performing a tile scan at $20 \mathrm{x}$ with a Z-stack of 7 slices and $2 \mu \mathrm{m}$ thickness. The images were stitched in the Zeiss Zen Blue program. We randomly selected 3-4 equal sized areas per tissue on the molecular layer and outlined the area of the dentate hilus in each mouse. The volume of the dentate hilus and molecular layer were calculated by multiplying their respective area by its thickness. The cell count was divided by the resultant section volume to obtain the total cell density in the dentate hilus and molecular layer per $\mathrm{mm}^{3}$.

\section{Statistical Analysis}

All assessments were performed by an observer blind to genotypes using GraphPad Prism 8.0 (GraphPad Software, La Jolla, CA, USA). One-way analysis of variance was performed on each data set followed by Tukey post hoc multiple comparisons with statistical significance reached at $\mathrm{P} \leq 0.05$.

\section{RESULTS}

\section{Genetic Deletion of sFRP3 Prevents Increased Astrogliosis Observed in Adult BubR $1^{\mathrm{H} / \mathrm{H}}$ Mice}

Reactive astrogliosis is defined by hypertrophic astrocytic morphology and an abnormal increase in the number of astrocytes. These phenotypes represent hallmarks of brain aging and agerelated neurodegeneration [22]. To investigate a possible neuroprotective effect of sFRP3 inhibition in age-related astrogliosis, immunohistological staining was performed with the astrocyte specific marker GFAP. Morphological image analysis revealed an increased intensity of GFAP immunoreactivity and hypertrophy observed in the molecular layer of the hippocampus in $B u b R 1^{\mathrm{H} / \mathrm{H}} ; s f r p 3 \mathrm{WT}$ mice compared to those in BubR1 WT;sfrp3 WT mice. However, this hypertrophic astrocytic morphology was ameliorated in $B u b R 1^{\mathrm{H} / \mathrm{H}}: s f r p 3 \mathrm{KO}$ mice (Fig. 1A). In addition to hypertrophic astrocytic morphology, our quantitative analysis showed a significant increase in the number of astroglia in the hippocampal hilus and molecular layer of $B u b R 1^{\mathrm{H} / \mathrm{H}}$ ;sfrp3 WT mice when compared to those in BubR1 WT;sfrp3 WT mice, which were reversed by genetic inhibition of sFRP3 (Fig. 1B). Notably, a reduction of sFRP3 expression in sfrp3 HET mice, which was reduced to about 50\% in WT littermates [12], was sufficient to prevent increased astrogliosis in $B u b R 1^{\mathrm{H} / \mathrm{H}}$ mice. Taken together, these results suggest that genetic inhibition of sFRP 3 can prevent reactive astrogliosis in $B u b R 1^{\mathrm{H} / \mathrm{H}}$ mice.

\section{Genetic Deletion of sFRP3 Prevents Increased Microglia Activation in Adult BubR ${ }^{\mathrm{H} / \mathrm{H}}$ Mice}

Microglia are the primary resident immune cells in the brain. During normal conditions, microglia are continuously surveilling their environment and facilitating the maintenance of synapses. However, these cells can be activated in response to a variety of stimuli during brain damage or cellular degeneration, and are thought to be the primary cells responsible for initiating the immune response to neurodegenerative pathology [23]. To explore a potential neuroprotective effect of sFRP3 inhibition on microglia, we performed immunohistological staining with 
BubR1 WT; sfrp3 WT
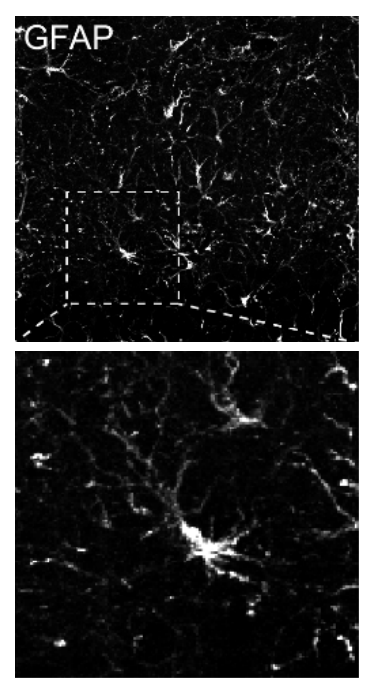

$B u b R 1^{\mathrm{H} / \mathrm{H}} ; \operatorname{sfp} 3 \mathrm{WT}$
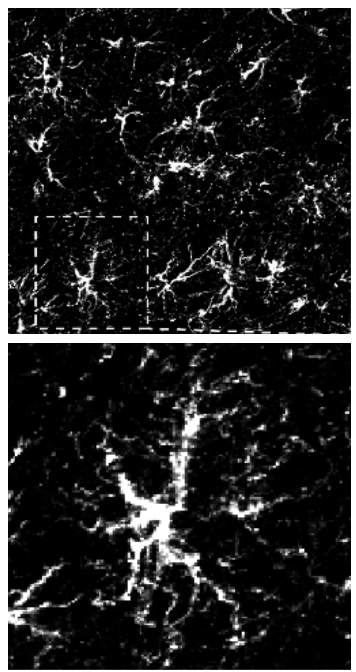

$B u b R 1^{\mathrm{H} / \mathrm{H}} ; \operatorname{sfp} 3 \mathrm{KO}$

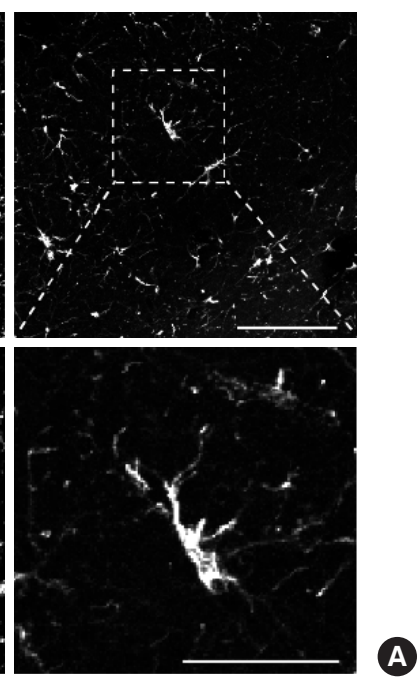

BubR1 WT; sfrp3 WT $(\mathrm{n}=5)$

$B u b R 1^{\mathrm{H} / \mathrm{H}} ; \operatorname{sfrp} 3 \operatorname{HET}(\mathrm{n}=4)$
$B u b R 1^{\mathrm{H} / \mathrm{H}} ; \operatorname{sfrp3} \mathrm{WT}(\mathrm{n}=5)$

$B u b R 1^{\mathrm{H} / \mathrm{H}} ; \operatorname{sfrp3} \mathrm{KO}(\mathrm{n}=5)$
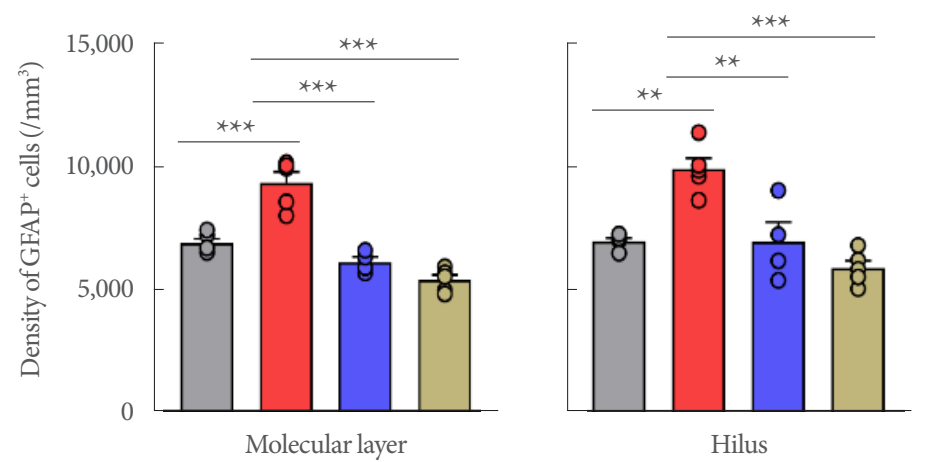

Hilus

Fig. 1. Genetic inhibition of sFRP3 prevents increased astrogliosis observed in $B u b R 1^{\mathrm{H} / \mathrm{H}}$ mice. (A) Representative confocal images of glial fibrillary acidic protein (GFAP) staining in the hilus of the dentate gyrus in each group. Scale bars: $50 \mu \mathrm{m}$ (above) and $20 \mu \mathrm{m}$ (below). (B) Stereological quantification of GFAP ${ }^{+}$astrocytes in the molecular layer and the hilus of the dentate gyrus of the hippocampus. Circles within each bar in graphs represent an individual mouse. Values represent mean \pm standard error of the mean. ${ }^{\star *} \mathrm{P}<0.01$, ${ }^{* * *} \mathrm{P}<0.001$, 1-way analysis of variance followed by Tukey post hoc corrections. All groups of mice at $8-10$ weeks of age were used at the time of initiating experiments. WT, wild-type; HET, heterozygous; KO, knockout.

the pan-microglia marker Iba1 in combination with CD68, a marker utilized to identify activated microglia. Confocal imaging and quantitative analysis of the hilus and molecular layer of the hippocampus did not reveal obvious morphological changes or quantitative differences in the number of $\mathrm{Iba}^{+}$microglia populations between the genotypes (Fig. 2A, B). However, colocalization analysis did identify an increased trend in $\mathrm{Ibal}^{+}$ $\mathrm{CD} 8^{+}$activated microglia populations in $\mathrm{BubR} 1^{\mathrm{H} / \mathrm{H}} ; s f r p 3 \mathrm{WT}$ mice compared to those in BubR1 WT;sfrp3 WT mice (Fig. 2A insets and 2B lower panels). More importantly, our colocaliza- tion analysis detected a significant decrease in $\mathrm{Iba}^{+} \mathrm{CD}^{+} 8^{+}$cells in the molecular layer of the hippocampus of $B u b R 1^{\mathrm{H} / \mathrm{H}} ; s f r p 3$ HET mice and $B u b R 1^{\mathrm{H} / \mathrm{H}} ; s f r p 3 \mathrm{KO}$ mice in comparison to $\mathrm{Bub}$ $R 1^{\mathrm{H} / \mathrm{H}} ; s f r p 3 \mathrm{WT}$ mice, suggesting that genetic inhibition of sFRP3 can successfully abolish age-related microglial reactivity to similar or below BubR1 WT;sfrp3 WT control levels (Fig. 2A insets and $2 \mathrm{~B}$ molecular layer lower panels). We similarly detected a significant decrease in age-related microglial reactivity in the hippocampal hilus of $B u b R 1^{\mathrm{H} / \mathrm{H}} ; s f r p 3 \mathrm{KO}$ mice when compared to $B u b R 1^{\mathrm{H} / \mathrm{H}} ;$ sfrp3 WT mice. Interestingly, when 




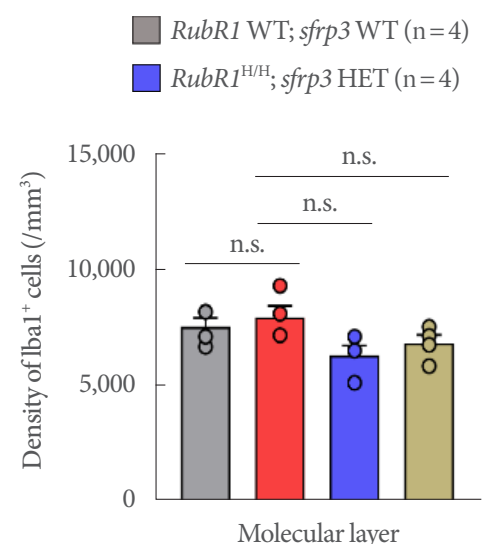

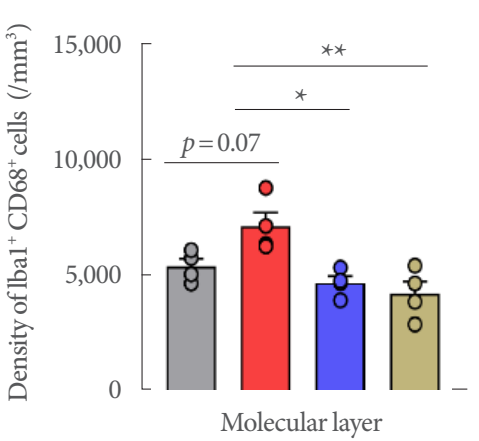

$R u b R 1^{\mathrm{H} / \mathrm{H}} ; \operatorname{sfrp} 3 \mathrm{WT}(\mathrm{n}=4)$

$R u b R 1^{\mathrm{H} / \mathrm{H}} ; \operatorname{sfrp} 3 \mathrm{KO}(\mathrm{n}=4)$

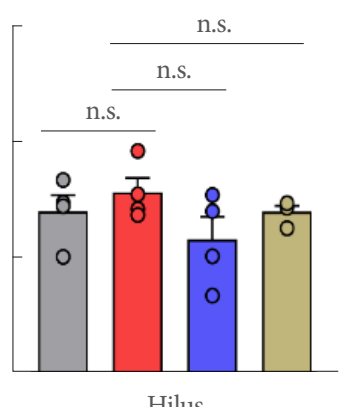

Hilus

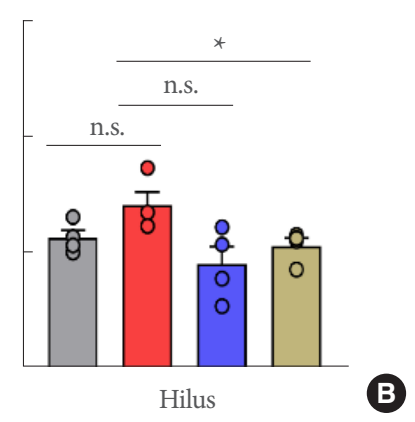

Fig. 2. Genetic inhibition of sFRP3 prevents increased microglia activation observed in $B u b R 1^{\mathrm{H} / \mathrm{H}}$ mice. (A) Representative confocal images of ionized calcium-binding adapter molecule 1 (Iba1) (green) and CD68 (red) staining in the hilus of the dentate gyrus in each group. Scale bars: $100 \mu \mathrm{m}$ (left) and $20 \mu \mathrm{m}$ (right). (B) Stereological quantification of $\mathrm{Iba1}^{+}$microglia and $\mathrm{Ibal}^{+} \mathrm{CD} 8^{+}$activated microglia in the molecular layer and the hilus of the dentate gyrus of the hippocampus. Circles within each bar in graphs represent an individual mouse. Values represent mean \pm standard error of the mean. ${ }^{*} \mathrm{P}<0.05,{ }^{* *} \mathrm{P}<0.01,1$-way analysis of variance followed by Tukey post hoc corrections. All groups of mice at 8-10 weeks of age were used at the time of initiating experiments. n.s., not significant; WT, wild-type; HET, heterozygous; KO, knockout.

compared to $B u b R 1^{\mathrm{H} / \mathrm{H}} ; s f r p 3 \mathrm{WT}$ mice, our analysis showed lower levels of $\mathrm{Iba}^{+} \mathrm{CD}^{+} 8^{+}$reactive microglia in the hippocampal hilus of $B u b R 1^{\mathrm{H} / \mathrm{H}} ; s f r p 3$ HET mice, although there was no statistical difference detected (Fig. 2B, hilus lower panels). This suggests that although partial genetic sFRP3 inhibition is effective in reducing age-related microglial reactivity, complete global sFRP3 deletion provides a more substantial attenuation of age-related microglial activation in the aging hippocampus (Fig. 2B, hilus lower panels). Collectively, these results suggest a preventative effect of sFRP3 inhibition to the endogenous microglia activation exhibited by $B u b R 1^{\mathrm{H} / \mathrm{H}}$ accelerated aged mice, which highlight that reductions in neuroinflammatory astrogliosis and microgliosis during aging may provide improvements in hippocampal BubR1 hypomorphic cellular dysfunctions associated with cognitive deficits.

\section{DISCUSSION}

The hippocampus is particularly vulnerable to aging and its dysfunction is implicated in age-related cognitive decline. However, the underlying molecular mechanism of why aging drives neurodegeneration and how this process can be reversed remains unknown. Previous studies from our group have shown that $B u b R 1^{\mathrm{H} / \mathrm{H}}$ mice exhibit impairments in hippocampal neurogenesis, oligodendrocyte development, and myelination that may lead to memory dysfunction [6-8].

Here we demonstrated increased astrogliosis in a $B u b R 1^{\mathrm{H} / \mathrm{H}}$ accelerated aging mouse model. In addition, we detected an increase in $\mathrm{Ibal}^{+} \mathrm{CD} 68^{+}$microglial reactivity as a result of accelerated aging due to BubR1 insufficiency. These results suggest that age-related endogenous neuroinflammation may be addi- 
tional contributors to hippocampal cellular impairments in aged mice, and genetic inhibition of sFRP3 in $B u b R 1^{\mathrm{H} / \mathrm{H}}$ mice reverse these aberrant biological processes. In addition to the neuroprotective role that SFRP3 inhibition confers toward restoring $B u b R 1^{\mathrm{H} / \mathrm{H}}$ mediated reductions in brain and body size, impaired neural progenitor proliferation, and myelination deficits [6-8], our current study expands on sFRP3-mediated inhibition of the Wnt signaling cascade, and its potential improvement on the endogenous neuroinflammatory and neurodegenerative characteristics of $B u b R 1^{\mathrm{H} / \mathrm{H}}$ mice. However, there are many unanswered questions that remain to be addressed. First, BubR1 insufficiency leads to increased reactive astrogliosis and microglia activation, suggesting a critical role for BubR1 in maintaining appropriate glial function. However, it remains unclear if increased neuroinflammatory processes are the main cause of cognitive impairments observed in $B u b R 1^{\mathrm{H} / \mathrm{H}}$ mice or just a pathological consequence resulting from BubR1 insuffciency. To address this critical question, the generation and use of BubR1 conditional $\mathrm{KO}$ mice to ablate protein expression in a cell type-specific fashion will be a valuable approach to provide further mechanistic insights by which BubR1 regulates agingrelated astrogliosis and microgliosis. Second, a number of reports have found that increased Wnt signaling activity can reverse cognitive dysfunction in a wide spectrum of neurological, neuropsychiatric and neurodegenerative disorders [10]. While our findings show that sFRP3 inhibition, which is known to increase Wnt/ $\beta$-catenin activity [12] has a beneficial impact on preventing neuroinflammation in $B u b R 1^{\mathrm{H} / \mathrm{H}}$ accelerated aging mice; it is of importance to determine whether the neuroprotective effects of sFRP3 inhibition may also be applicable to other cognitive disorders. If this is the case, sFRP3 inhibition could be a general therapeutic strategy to delay and/or prevent the onset of cognitive decline associated with many brain disorders. Lastly, whether and how declining BubR1 levels alter sFRP3 expression to affect hippocampal function has never been explored. Therefore, elucidating the relationship between BubR1 and Wnt signaling contributions to the aging hippocampal phenotypes observed in this study will be critical to identify a new mechanistic link between neuroinflammation and aging. Additional research is warranted as this remains a key open question.

In conclusion, the present study demonstrates a new and essential role for sFRP3 inhibition in preventing endogenous neuroinflammatory gliosis exhibited by $B u b R 1^{\mathrm{H} / \mathrm{H}}$ accelerated aging mice. Given that neuroinflammation and neurodegenera- tion are pathological mechanisms that underlie age-related cognitive disability, our study identifies that sFRP3 inhibition plays a neuroprotective role in neuroinflammation, thus providing a potentially novel investigative therapeutic strategy in age-related degenerative pathology.

\section{ACKNOWLEDGEMENTS}

We would like to thank Dr. Jan M. van Deursen at Mayo Clinic for kindly providing $B u b R 1^{\mathrm{H} / \mathrm{H}}$ mice, and Dr. Jeremy Nathans at Johns Hopkins University for sFRP3 KO mice.

\section{AUTHOR CONTRIBUTION STATEMENT}

- Conceptualization: $A M C R, M D, A O, M H J$

- Data curation: $A M C R, M D, K H Y$

- Formal analysis: $A M C R, M D$

- Funding acquisition: $M H J$

- Methodology: $A M C R, M D, K H Y$

- Project administration: $M H J$

- Visualization: $A M C R, M D$

- Writing-original draft: $A M C R, M H J$

- Writing-review \& editing: $A M C R, M H J, A O$

\section{REFERENCES}

1. Stern Y. Cognitive reserve in ageing and Alzheimer's disease. Lancet Neurol 2012;11:1006-12.

2. García-Castillo H, Vásquez-Velásquez AI, Rivera H, Barros-Núñez P. Clinical and genetic heterogeneity in patients with mosaic variegated aneuploidy: Delineation of clinical subtypes. Am J Med Genet A 2008;146A:1687-95.

3. Matsuura S, Matsumoto Y, Morishima K, Izumi H, Matsumoto H, Ito E, et al. Monoallelic BUB1B mutations and defective mitoticspindle checkpoint in seven families with premature chromatid separation (PCS) syndrome. Am J Med Genet A 2006;140:358-67.

4. Baker DJ, Dawlaty MM, Wijshake T, Jeganathan KB, Malureanu L, van Ree, JH, et al. Increased expression of BubR1 protects against aneuploidy and cancer and extends healthy lifespan. Nat Cell Biol 2013;15:96-102.

5. Baker DJ, Jeganathan KB, Cameron JD, Thompson M, Juneja S, Kopecka A, et al. BubR1 insufficiency causes early onset of agingassociated phenotypes and infertility in mice. Nat Genet 2004;36: 744-9.

6. Yang Z, Jun H, Choi CI, Yoo KH, Cho CH, Hussaini SMQ, et al. 
Age-related decline in BubR1 impairs adult hippocampal neurogenesis. Aging Cell 2017;16;598-601.

7. Choi CI, Yoo KH, Hussaini SM, Jeon BT, Welby J, Gan H, et al. The progeroid gene BubR1 regulates axon myelination and motor function. Aging 2016;8;2667-88.

8. Cho CH, Yang Z, Yoo KH, Oliveros A, Jang MH. BubR1 insufficiency impairs affective behavior and memory function in mice. Int Neurourol J 2018;22(Suppl 3);S122-30.

9. Komiya Y, Habas R. Wnt signal transduction pathways. Organogenesis 2008;4:68-75.

10. Hussaini SM, Choi CI, Cho CH, Kim HJ, Jun H, Jang MH. Wnt signaling in neuropsychiatric disorders: Ties with adult hippocampal neurogenesis and behavior. Neurosci Biobehav Rev 2014;47: 369-83.

11. Inestrosa NC, Varela-Nallar L. Wnt signaling in the nervous system and in Alzheimer's disease. J Mol Cell Biol 2014;6:64-74.

12. Jang MH, Bonaguidi MA, Kitabatake Y, Sun J, Song J, Kang E, et al. Secreted frizzled-related protein 3 regulates activity-dependent adult hippocampal neurogenesis. Cell Stem Cell 2013;12:215-23.

13. Jang MH, Kitabatake Y, Kang E, Jun H, Pletnikov MV, Christian $\mathrm{KM}$, et al. Secreted frizzled-related protein 3 (sFRP3) regulates antidepressant responses in mice and humans. Mol Psychiatry 2013; 18:957-8.

14. Seib DR, Corsini NS, Ellwanger K, Plaas C, Mateos A, Pitzer C, et al. Loss of Dickkopf-1 restores neurogenesis in old age and coun- teracts cognitive decline. Cell Stem Cell 2013;12:204-14.

15. García-Cáceres C, Balland E, Prevot V, Luquet S, Woods SC, Koch $\mathrm{M}$, et al. Role of astrocytes, microglia, and tanycytes in brain control of systemic metabolism. Nat Neurosci 2019;22:7-14.

16. Bolós M, Perea JR, Avila J. Alzheimer's disease as an inflammatory disease. Biomol Concepts 2017;18:37-43.

17. Palmer AL, Ousman SS. Astrocytes and aging. Front Aging Neurosci 2018;10:337.

18. Hussaini SMQ, Jang MH. New roles for old glue: astrocyte function in synaptic plasticity and neurological disorders. Int Neurourol J 2018;22(Suppl 3):S106-14.

19. Hartman TK, Wengenack TM, Poduslo JF, van Deursen JM. Mutant mice with small amounts of BubR1 display accelerated age-related gliosis. Neurobiol Aging 2007;28:921-7.

20. Cho CH, Yoo KH, Oliveros A, Paulson S, Hussaini SMQ, van Deursen JM, et al. sFRP3 inhibition improves age-related cellular changes in BubR1 progeroid mice. Aging Cell 2019;18:e12899.

21. Hussaini SM, Jun H, Cho CH, Kim HJ, Kim WR, Jang MH. Heatinduced antigen retrieval: an effective method to detect and identify progenitor cell types during adult hippocampal neurogenesis. J Vis Exp 2013;78:50769.

22. Sofroniew MV. Astrogliosis. Cold Spring Harb Perspect Biol 2014; 7:a020420

23. Hickman S, Izzy S, Sen P, Morsett L, El Khoury J. Microglia in neurodegeneration. Nat Neurosci 2018;21:1359-69. 